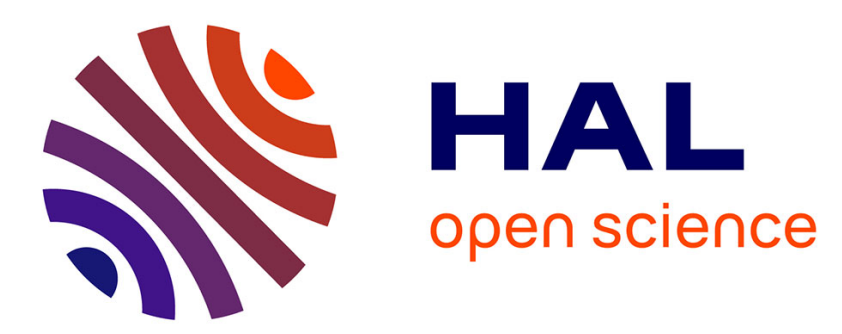

\title{
Experimental and numerical analysis of the flow inside a configuration including an axial pump and a tubular exchanger
}

\author{
Moises Solis, Florent Ravelet, Sofiane Khelladi, Farid Bakir
}

\section{To cite this version:}

Moises Solis, Florent Ravelet, Sofiane Khelladi, Farid Bakir. Experimental and numerical analysis of the flow inside a configuration including an axial pump and a tubular exchanger. Proceedings of ASME 2010 3rd Joint US-European Fluids Engineering Summer Meeting and 8th International Conference on Nanochannels, Microchannels, and Minichannels, FEDSM2010-ICNMM2010., Aug 2010, Montreal, Canada. pp.FEDSM-ICNMM2010-30723， 10.1115/FEDSM-ICNMM2010-30723 . hal-00505772

\section{HAL Id: hal-00505772 \\ https://hal.science/hal-00505772}

Submitted on 3 Sep 2012

HAL is a multi-disciplinary open access archive for the deposit and dissemination of scientific research documents, whether they are published or not. The documents may come from teaching and research institutions in France or abroad, or from public or private research centers.
L'archive ouverte pluridisciplinaire HAL, est destinée au dépôt et à la diffusion de documents scientifiques de niveau recherche, publiés ou non, émanant des établissements d'enseignement et de recherche français ou étrangers, des laboratoires publics ou privés. 


\section{EXPERIMENTAL AND NUMERICAL ANALYSIS OF THE FLOW INSIDE A CONFIGURATION INCLUDING AN AXIAL PUMP AND A TUBULAR EXCHANGER}

Moisés Solis, Florent Ravelet a) Sofiane Khelladi, and Farid Bakir

Arts et Metiers ParisTech, DynFluid, 151 boulevard de l'Hôpital, 75013 Paris, France.

(Dated: Proceedings of 3rd Joint US-European Fluids Engineering Summer Meeting,) (August 1-5 2010, Montreal, Canada, FEDSM-ICNMM2010-30723)

In centrifugal and axial pumps, the flow is characterized by a turbulent and complex behavior and also by physical mechanisms such as cavitation and pressure fluctuations that are mainly due to the strong interactions between the fixed and mobile parts and the operating conditions. These fluctuations are more important at the tip clearance and propagate upstream and downstream of the rotor. The control of the fluctuating signal amplitudes can be achieved by incrementing the distance between the components mentioned above. This paper presents experimental and numerical results concerning the operation of a configuration that includes an axial pump and a bundle of tubes that mimics the cool source of a heat exchanger. The pump used in the tests has a low solidity and two blades designed in forced vortex, the tip clearance is approximately $3.87 \%$ of tip radius. The experimental measures were carried out using a test bench built for this purpose at the DynFluid Laboratory which was accomodated conveniently with a variety of instruments. Firstly, the characteristic curves were drawn for the pump at $1500 \mathrm{rpm}$ and then a set of measurements concerning the use of pressure sensors was done in order to recover for different flow rates the static pressure signals upstream and downstream the pump and the exchanger. The pressure fluctuations and the performance curve were compared to the numerical results. The numerical simulations were carried out by using a Fluent code, the URANS (Unsteady Reynolds Averaged Navier-Stokes) approach and the k- $\omega$ SST turbulence model were applied to solve the unsteady, incompressible and turbulent flow. To record the fluctuating pressure signal, virtual sensors were necessary and placed at the same positions as in the experiments.

Keywords: Axial Pump, Pressure Fluctuations, Cavitation, Tubular Exchanger, Test Bench

\section{NOMENCLATURE}

\begin{tabular}{lll}
\multicolumn{3}{l}{ Roman characters } \\
$c$ & {$[\mathrm{~mm}]$} & Chord length \\
$F$ & {$[\mathrm{~Hz}]$} & frequency \\
$H$ & {$[\mathrm{~m}]$} & Static head and static head at nominal point \\
$p$ & {$[\mathrm{~mm}]$} & $2 \pi r / z$, circumferential distance \\
$P$ & {$[\mathrm{~Pa}]$} & Pressure \\
$Q$ & {$\left[\mathrm{~m}^{3} / \mathrm{h}\right]$} & Flow rate \\
$r$ & {$\left[\mathrm{~mm}^{2}\right.$} & Radius \\
$s$ & {$[-]$} & $p / c$, Solidity
\end{tabular}

Greek characters

$\beta \quad\left[{ }^{o}\right] \quad$ Blade angle

$\begin{array}{lll}\delta_{t} & {[\mathrm{~mm}]} & \text { Tip clearance }\end{array}$

$\eta \quad[-] \quad$ Hydraulic efficiency

$\rho \quad\left[\mathrm{kg} \cdot \mathrm{m}^{-3}\right]$ Density

Subscripts

$1 \quad$ [-] Inlet

$B P \quad[-] \quad$ Blade passage

eff [-] Effective

$h \quad$ [-] $\quad$ Blade hub

midspan [-] $50 \%$ of blade span

$\begin{array}{lll}N & \text { [-] Nominal }\end{array}$

$R \quad$ [-] Rotational

ref [-] Reference

$t \quad[-] \quad$ Blade tip

\footnotetext{
a)Electronic mail: florent.ravelet@ensta.org
}

\section{INTRODUCTION}

The design of an efficient, quiet and reliable axial-flow pump is a challenge for the designers nowadays. The main difficulty is the existence of various geometrical parameters that have a direct influence on the hydraulic performance. A solution to this problem is to analyze and better understand of the flow field inside the rotating and stationary parts with the help of numerical simulations and experimental tests. It is well known that an axial pump generates a complex flow field which is characterized by the presence of physical mechanisms and pressure fluctuations. In this kind of pump configuration, the relative motion between the rotating and stationary part causes strong interactions that are mainly associated to the blade passing frequency and are more important close to the tip clearance in the case of axial pumps and close to the tongue for centrifugal pumps. A potential reduction of these interactions can be achieved by varying the distance between the fixed and the mobile parts of the configuration.

Many research works have been done in the domain of axial pumps covering the cavitation, pressure fluctuations and design methods. The influence of the tip clearance was reported by Mitchell [1] who investigated experimentally an axial pump. This work pointed out the existence of a critical tip clearance below which surface cavitation at the blade tip occurs first and above which 
vortex cavitation due to the clearance flow occurs first. Laborde et al. [2] found an optimum clearance geometry to eliminate clearance cavitation through the study of cavitation patterns under different operations and geometrical conditions. The influence of these cavitation patterns were determined by cavitation tests on an axial flow pump by Horie et al. [3]. Over a wide range of operating conditions, a little change of pump head was observed when the cavitation on the blade surface was developed considerably. Others researchers analyzed the effects of guide vanes and rear stator. Kaya [4] experimentally studied two different axial flow pump impellers with and without guide vanes. Using guide vanes, the total pump efficiency was increased by about $3 \%$. Gao et al. [5] investigated the effects of including a rear stator on flow fields using for his studies two water-jet pumps with and without a rear stator. They found a good agreement with the experimental results with improvement on the static pressure and the efficiency.

Not much work has been done to establish a relationship between cavitation development and the pressure fluctuations of an axial-flow pump. Saito [6] investigated the change in pressure fluctuations spectrum due to cavitation and pointed out that it mainly depends on the tip clearance cavitation development. Fu-jun et al. [7] analyzed the flow in axial-flow pump through transient simulations and large eddy simulation (LES), obtaining the pressure fluctuations under various operation conditions. The maximum amplitude takes place at the inlet of impeller and they increases from hub to tip, but decreases at the middle area. The fluctuation becomes stronger as the flow rate is far from optimum operation point.

Another important factor of an axial pump is the blade design. Vad et al. [8] numerically compared two axialflow pump rotors designed in free vortex and non-free vortex design having identical basic geometrical and flow rate parameters. He concluded that non-free vortex design is an efficient method to increase the specific performance but as a drawback, the risk of cavitation also increases. On the other hand, for the non-free vortex rotor the efficiency drops more intensely with increase of tip clearance and the noise level may be lower than for the free vortex rotor. A similar work was done by Ida [9] with experiments on a forced-vortex impeller in an axial flow fan without inlet vanes, assuming that the axial velocity component is uniformly distributed from hub to tip.

Numerical calculations using CFD codes are an important tool to accurately predict the axial pump behavior. Miner [10] used a commercial code to compute the flow field within the first stage impeller of a two stage axial flow pump, showing that the numerical results closely matched the shapes and magnitudes of the measured profiles. Other examples are the works of DeSheng et al. [11] who simulated using Fluent code the flow field of a high efficiency axial-flow pump. He found that the static pressure on pressure side of rotor blades increases slightly at radial direction, and remains almost constant in circum- ferential direction at design conditions, while it increases gradually from inlet to exit on suction side along the flow direction. The experimental results showed that the inlet flow is almost axial and the prerotation is very small at design conditions.

Concerning the configuration formed by an axial pump and a tubular heat exchanger, the understanding of the flow behavior does not seem to be clear especially when the analysis is focused on the pressure fluctuations and the cavitation in the region upstream of the exchanger. The current paper presents an experimental and numerical investigation of the interaction between an axial pump and a bundle of tubes that mimics the cool source of a heat exchanger. The experimental measurements were the static head rise, the pressure fluctuations upstream and downstream the rotor for different flow rates. The axial flow pump used for the experiments was a particular rotor of low solidity, two blades and the tip clearance is about $3.87 \%$ of tip radius. On the other hand, numerical simulations were carried out using the URANS (Unsteady Reynolds Averaged Navier-Stokes) approach and the k- $\omega$ SST turbulence model were applied to solve the unsteady, incompressible and turbulent flow. In the unsteady calculations, virtual sensors were used to recover the fluctuating pressure signal at the same positions as in the experiments.

\section{GEOMETRICAL CONFIGURATION AND OPERATING CONDITIONS}

For this study, the configuration is based on an axialflow pump and a bundle of tubes hereafter designated by the expression "tubular exchanger" though no heat is actually exchanged in the reported works. It can be placed at several distances downstream of the pump (see figure 1) in order to study the influence of this distance on the level of pressure fluctuations. In this Paper, results for only one position are reported.

The test loop was conditioned with some instruments positioned conveniently to measure the static pressure, flow rate, electric power and pressure fluctuations. The rotor used has a particular geometry and low solidity. It has two blades designed in forced vortex and presents an important tip clearance. The main geometrical characteristics of the reference pump and flow parameters are summarized in table 1.

\section{COMPUTATIONAL DOMAIN AND NUMERICAL PROCEDURE}

In order to compare the numerical and experimental results, the domain for the calculations was obtained from the original geometries of the test bench used during the experimental measurements. The calculation domain extends from the suction and discharge pipe (see figure 2 ). To avoid any influence of boundary limits, they were 


\begin{tabular}{cc}
\hline Rotor & \\
\hline Design method & forced vortex \\
Total blades number, $z$ & 2 \\
Tip radius, $r_{t}$ & $91.45 \mathrm{~mm}$ \\
Hub radius, $r_{h}$ & $27.61 \mathrm{~mm}$ \\
Hub-to-tip ratio, $r_{t} / r_{h}$ & 0.30 \\
Tip clearance, $\delta_{t}$ & $3.87 \%$ of $r_{t}$ \\
Blade profile & NACA \\
Blade solidity tip, $s_{t}$ & 0.481 \\
Blade solidity hub, $s_{h}$ & 0.843 \\
Blade solidity midspan, $s_{m i d s p a n}$ & 0.414 \\
Tip blade angle, $\beta_{t 1}$ & $14.36^{\circ}$ \\
Hub blade angle, $\beta_{h 1}$ & $14.71^{\circ}$ \\
Midspan blade angle, $\beta_{\text {midspan }}$ & $15.87^{\circ}$ \\
\hline Bundle of tubes & \\
\hline Holes number & 37 \\
Holes diameter & $14.2 \mathrm{~mm}$ \\
Holes depth & $200 \mathrm{~mm}$ \\
\hline Operating Conditions & \\
Nominal flow rate, $Q_{N}$ & $145 \mathrm{~m} / \mathrm{h}$ \\
Nominal static head, $H_{N}$ & $0.83 \mathrm{~m}$ \\
Rotational speed, $\mathrm{N}$ & $1500 \mathrm{rpm}$
\end{tabular}

TABLE I. Rotor and "tubular exchanger" geometrical characteristics and operating conditions

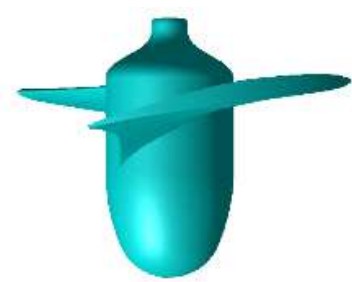

(a) Axial flow pump

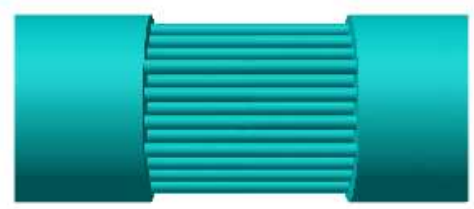

(b) Tubular Exchanger
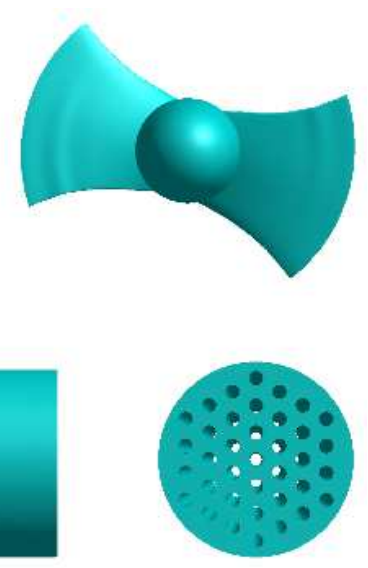

.

IG. 1. Geometry of the configuration including an axial pump and exchanger

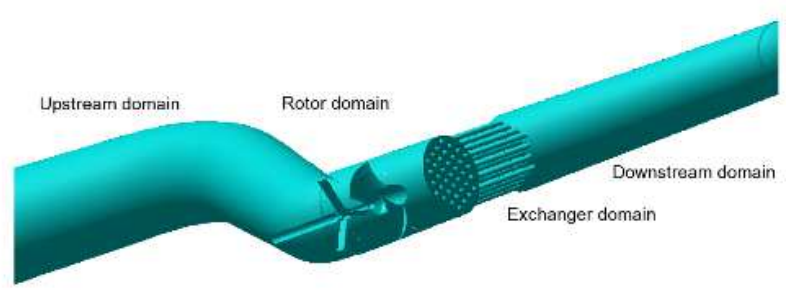

Figure 2. Computational domain.

FIG. 2. Computational domain appropriately extended to let the flow develop, ensuring numerical stability and minimizing boundary conditions effects. On the other hand, it is clear that the presence of complex geometries such as the rotor and the aspiration components will make the mesh hybrid. So, the entire domain was divided into various sub-domains. Thus, the regions considered are the suction pipe, rotor, tip clearance, tubular exchanger and discharge pipe. For the parts before the rotor and after the exchanger, the mesh types is a mixture of tetrahedral and hexahedral elements. Concerning the fluid volume of the rotor, the mesh was refined around the blades and the elements were tetrahedral. In the case of the tip clearance which has the shape of a ring, the elements considered were hexahedral following the flow direction, with 20 cells in the radial direction. The exchanger region is modeled by equidistant hexahedral cells distributed in the $\mathrm{z}$ direction. A mesh independency study was not made because of the significant number of components, but the retained mesh size was considered sufficient and its distribution adequate to validate the final results. The flow modeling was achieved by Fluent code based on the finites volumes method. The equations governing the fluid such as the continuity, momentum and turbulence equations were solved for the steady calculations in the moving reference frame and the sliding mesh technique was applied for the unsteady calculations, allowing to simulate the three-dimensional flow inside the pump and the interactions between the rotor and the external casing. The turbulence effects was simulated by using the k- $\omega$ SST model and the boundary limits imposed are an uniform normal velocity at inlet and the outflow condition at the outlet domain. The time dependent term scheme was a second order implicit. The pressure-velocity coupling is performed using the SIMPLE algorithm "Semi-Implicit Method for Pressure Linked equations". Second order, upwind discretization is used for convection terms and central difference schemes for diffusion terms. The time step for the unsteady calculations has been set to 1.104 $\mathrm{s}$ that will allow to have a good resolution in time and a good frequency resolution. Please note that it is also the experimental data acquisition rate. The simulations are carried out by doing a steady simulation to construct the performances curves and using then the output as an initial condition for the unsteady simulations. To investigate local boundary layer variables, the mesh used in the rotor is not the most adequate given the complexity of the geometry, but the global ones are well calculated.

Thus, approximately 2.5 million cells were used for the steady and unsteady calculations which will be validated by the experimental results. The table 2 summarizes the cells number employed for each region of the complete configuration considered for the modeling and the figure 3 shows some details of the grid on the rotor and the exchanger. On the other hand, a relatively large amount of time steps are required to pass the transient and to achieve a stable periodic solution. Virtual sensors were placed in the same positions as they were on the test 


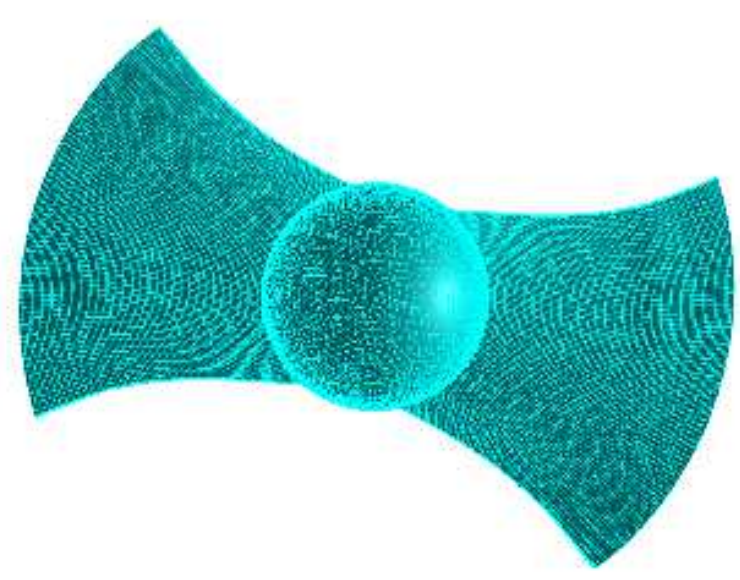

(a) Rotor mesh

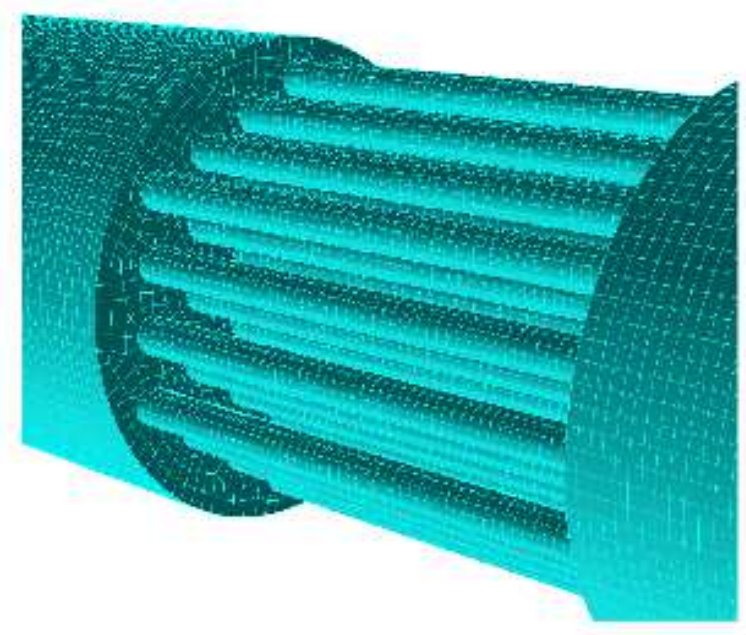

(b) Tubular exchanger mesh

FIG. 3. Meshes of the rotor and exchanger domain

facility to record time series of the static pressure. The data are analysed in the frequency domain using the Fast Fourier Transform.

\begin{tabular}{cc}
\hline Domain & Cells number \\
\hline Suction pipe & 425621 \\
Rotor & 1560793 \\
Tip Clearance & 208860 \\
Exchanger & 45954 \\
Discharge pipe & 331292 \\
\hline Total & 2572520 \\
\hline
\end{tabular}

TABLE II. Total cells number for the configuration axial pump and exchanger

\section{EXPERIMENTAL FACILITY}

The experimental tests were carried out at the DynFluid laboratory. For this purpose, an experimental test

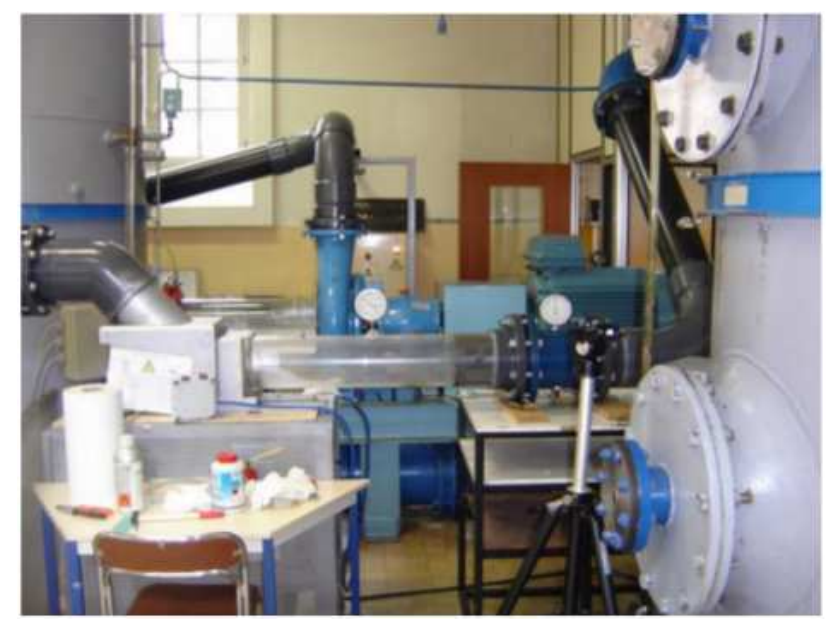

FIG. 4. Scheme of the DynFluid axial pump test-ring

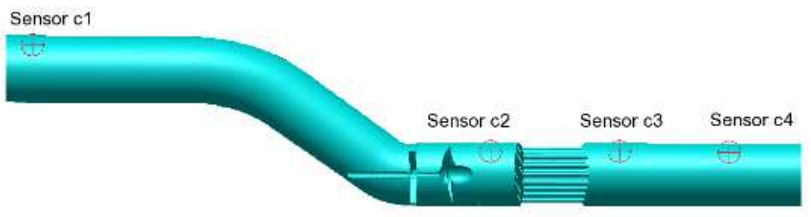

Figure 5. Pressure sensor positions in the test facility.

FIG. 5. Pressure sensor positions in the test facility

facility was designed and adapted to the existing installation which is composed of two independent but interconnected loops: one for centrifugal pumps and another for axial pumps. The test bench is shown in figure 4 . The axial-flow pump loop used for this investigation consists of:

- Two storage tanks with a capacity of $4 \mathrm{~m}^{3}$ that can be filled and emptied using two electrical butterfly control valves that are also used to adjust the flow rate accurately.

- A liquid ring vacuum pump that controls the pressure at the free surface inside the two storage tanks.

- An electromagnetic KROHNE flowmeter placed downstream of the axial pump (accuracy \pm 0.5 $\left.\mathrm{m}^{3} / \mathrm{h}\right)$.

- A $22 \mathrm{~kW}$ alternative motor powered by a frequency inverter to drive the tested axial pump at different rotational speeds (accuracy $\pm 0.05 \mathrm{~kW}$ ).

- A magnetic tachometer to measure the rotational speed (accuracy 0.1\%).

- A transparent acrylic cover in the zone of the axial pump to observe the flow and others aspects like the cavitation development.

- A centrifugal pump to drive the fluid toward the axial pump when it is necessary. It is situated about 
50 pipe diameter upstream of the axial pump, and is mounted between two DilatoFlex elements in order to attenuate the propagation of pressure fluctuations.

- Metallic differential manometers backed up by piezoresistive pressure sensor (accuracy $\pm 3 \mathrm{mBars}$ ).

- A temperature probe (accuracy 1\%): the average temperature during the tests was $18^{\circ}$.

- Four pressure sensor PCB Piezotronics (Model 106B51) that are flush-mounted and placed at various positions upstream and downstream of the rotor (see details in figure 5).

i) Sensor c1 positioned approximately at a distance $119.6 \mathrm{~cm}$ from the rotor (leading edge).

ii) Sensor c2 positioned approximately at a distance $14.4 \mathrm{~cm}$ from the rotor (leading edge).

iii) Sensor c3 positioned approximately at a distance $54.4 \mathrm{~cm}$ from the rotor (leading edge).

iv) Sensor c4 positioned approximately at a distance $88.9 \mathrm{~cm}$ from the rotor (leading edge).

\section{EXPERIMENTAL AND NUMERICAL RESULTS GLOBAL PERFORMANCES CURVES}

The first series of measurements concern the overall performances at $1500 \mathrm{rpm}$ for the configuration without the tubular exchanger. For this purpose, experimental and numerical tests were carried out to determine the operating point. The static pressure measurements were made at a distance upstream of the rotor (leading edge) of $132.1 \mathrm{~cm}$ and a distance downstream of the rotor (leading edge) of $116.9 \mathrm{~cm}$. Figure 6 presents the global stationary pump performances compared to the experimental measurements. For the $\mathrm{Q} \mathrm{H}$ curve the trend is the same for the numerical and experimental results, but the experimental curve is approximately $0.2 \mathrm{~m}$ higher than the numerical curve. Concerning the hydraulic efficiency, there is a very good agreement for flow rates below 150 $\mathrm{m}^{3} / \mathrm{h}$. Above this flow rate, there is a strong discrepancy which will have an influence on the determination of the nominal point. Thus, the numerical nominal point does not match with the experimental one. The numerical point is $145 \mathrm{~m}^{3} / \mathrm{h}$ and $0.83 \mathrm{~m}$ and the experimental one is $210 \mathrm{~m}^{3} / \mathrm{h}$ and $0.54 \mathrm{~m}$. The differences can be explained by the lack of precision on the electric power measurement and differential pressure. Other reason of the static pressure difference would be that the rotor geometry is particular and differs from that obtained by the manufacturing process. Please also note the very peculiar geometry of the pump, with low solidity and high stagger angle. Visualisation using cavitation have confirmed that the flow is adapted at a flow rate of $210 \mathrm{~m}^{3} / \mathrm{h}$ in the experiment.

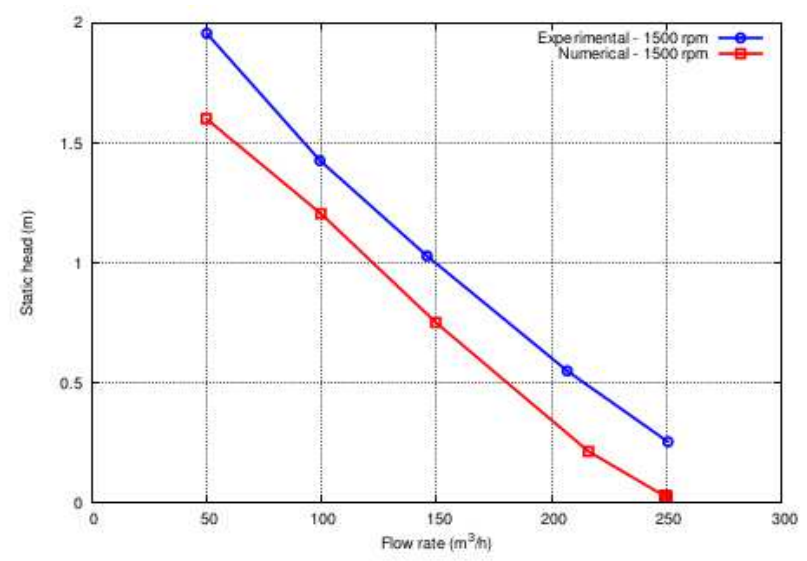

(a) Q-H

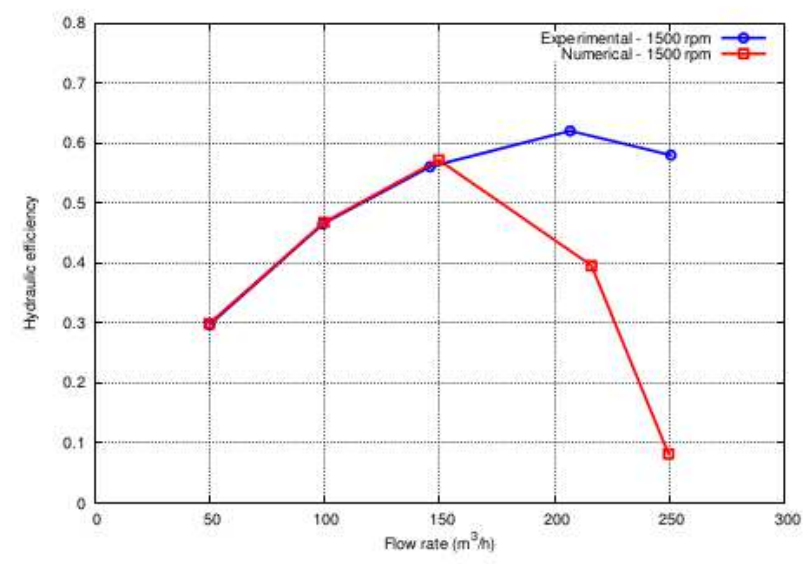

(b) Q- $\eta$

FIG. 6. Comparison between the numerical and experimental results of characteristics curves

\section{PRESSURE FLUCTUATIONS}

A series of measurements was performed for the configuration with axial pump and the other including the pump and the tubular exchanger. Concerning the single pump, three flow rates $\left(50 \mathrm{~m}^{3} / \mathrm{h}, 100 \mathrm{~m}^{3} / \mathrm{h}\right.$ and 145 $\mathrm{m}^{3} / \mathrm{h}$ ) were tested and information about pressure signals were recorded and compared. In the tests, four pressure sensors (c1, c2, c3 and c4) were distributed upstream and downstream of the rotor (see figure 5 for details). The figure 7 shows the comparison of frequency spectra obtained from the four sensors at different flow rates. The first impression of the results reveals that the spectra are different between the pressure sensors. There is a small peak at $F_{R}=25 \mathrm{~Hz}$ that could be linked to a small unbalance in the system. The main peak is at the blade passing frequency $F_{B P}=50 \mathrm{~Hz}$ and the analysis is focused on this peak. Its amplitude is high at inlet pump (sensor c1), but it decreases as we move away from the pump (sensor c4) at any flow rate. The apparition of peaks at frequencies lower than $F_{B P}$ is due to the centrifugal pump which was used to ensure the flow rate to 

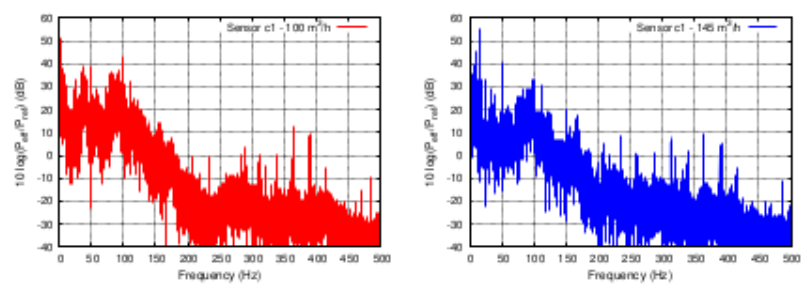

(a) Sensor cl
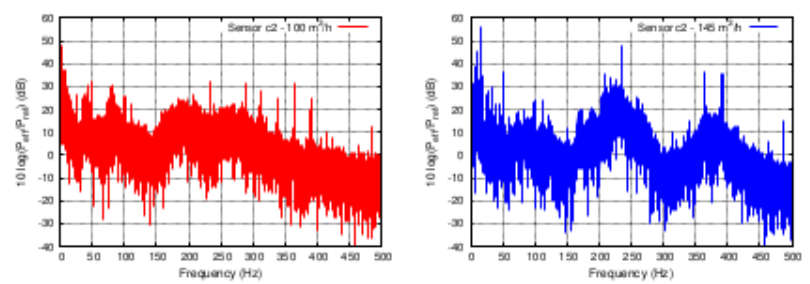

(b) Sensor c2
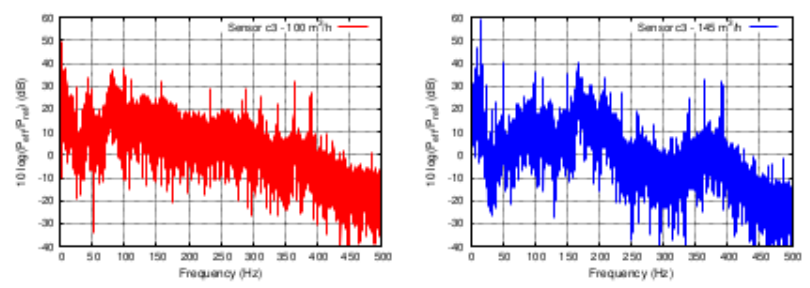

(c) Sensor c3
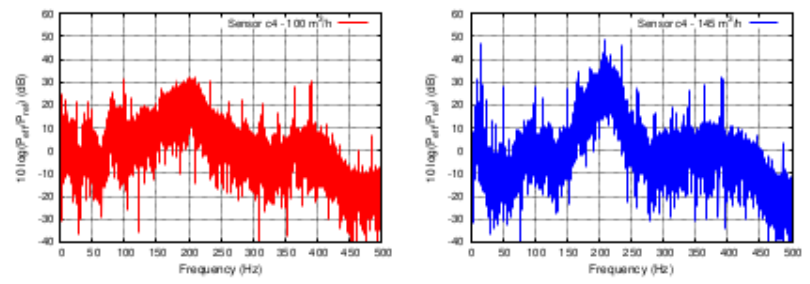

(d) Sensor c4

FIG. 7. Comparison of pressure frequency spectrum for the single pump. $P_{r e f}=1 \mathrm{~Pa}$

the axial pump. On the other hand, it can be seen that for the four sensors $\mathrm{c} 1, \mathrm{c} 2, \mathrm{c} 3$ and $\mathrm{c} 4$ the fluctuations increase when the flow rate begins to rise from $100 \mathrm{~m}^{3} / \mathrm{h}$ to $145 \mathrm{~m}^{3} / \mathrm{h}$.

The influence of inlet conditions were studied for the configuration single pump in order to determine the importance of pressure inlet. So, some measurements of pressure for a flow rate of $92 \mathrm{~m}^{3} / \mathrm{h}$ were made and compared when the axial pump works with the centrifugal pump and when it works alone. In this case, the flow rate mentioned was the maximum we could obtain from the circuit with the axial pump operating. The inlet pressure without centrifugal pump was approximately $-105 \mathrm{mBar}$ and with centrifugal pump it was $-30 \mathrm{mBar}$. From the figure 8 , which was obtained by a FFT analysis, we can point out specially at $F_{B P}$ some differences in relation to pressure amplitudes. In most of sensors except sensor c2, the amplitudes are lower when the centrifugal pump
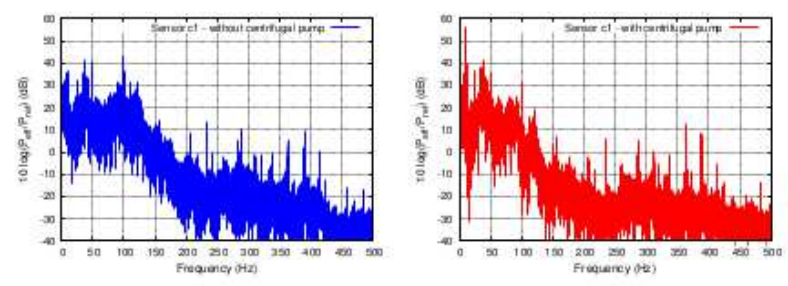

(a) Sensor $\mathrm{cl}$
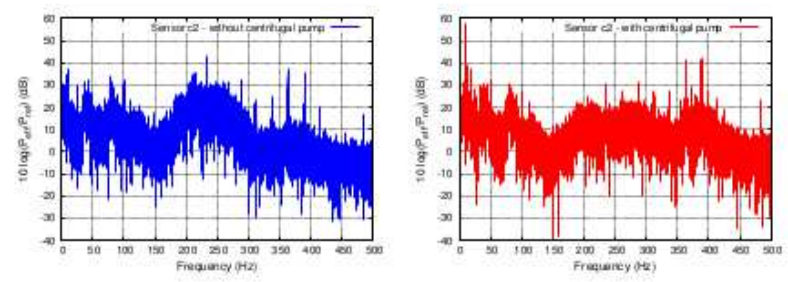

(b) Sensor c2
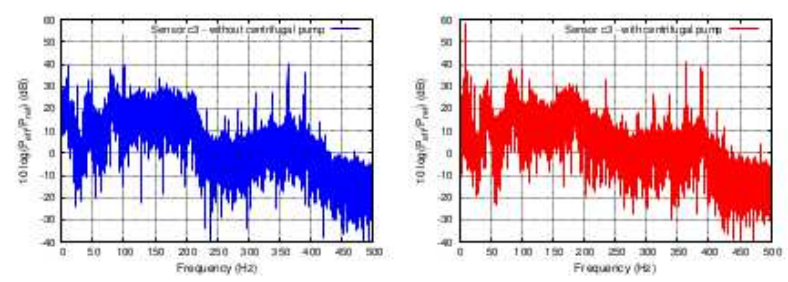

(c) Sensor c3
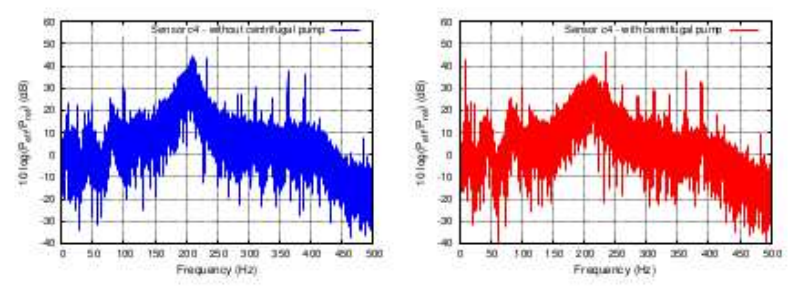

(d) Sensor c4

FIG. 8. Comparison of pressure frequency spectrum for the single pump at $92 \mathrm{~m}^{3} / \mathrm{h}$ with different suction conditions. $P_{\text {ref }}=1 \mathrm{~Pa}$

is operating in this way varying the suction pressure and the turbulence intensity. Thus, the importance of inlet conditions is manifested by the pressure fluctuations results that could be considered when performing experimental tests and secondly a solution to decrease pressure fluctuations instead of having to do modifications to the geometry.

In order to establish a relation between pressure fluctuations and the position of the exchanger. Two positions were tested, position 1 which corresponds when the exchanger is near to the pump (exchanger between sensor $\mathrm{c} 2$ and $\mathrm{c} 3$ ) and position 2 when the exchanger is far from the pump (exchanger between sensor c 3 and $\mathrm{c} 4$ ). The exchanger was fixed adequately to prevent movement during operation of the circuit. The flow rates tested were two $\left(145 \mathrm{~m} 3 / \mathrm{h}\right.$ and $\left.210 \mathrm{~m}^{3} / \mathrm{h}\right)$. First, considering a flow rate of $145 \mathrm{~m}^{3} / \mathrm{h}$ (see figure 9), the spectra indicate a reduction of amplitudes in sensor c1 placed upstream the 

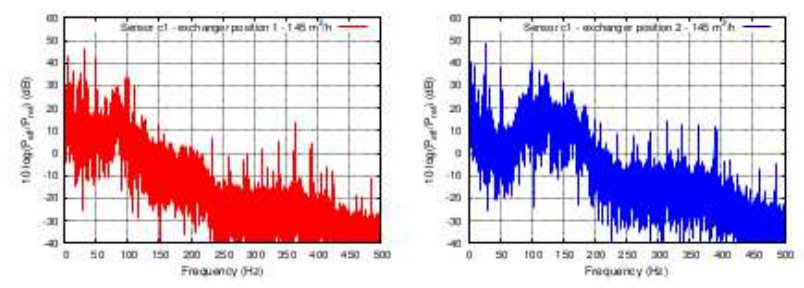

(a) Sensor $\mathrm{cl}$
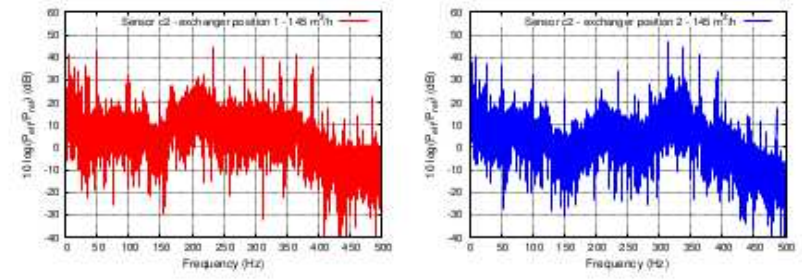

(b) Sensor c2
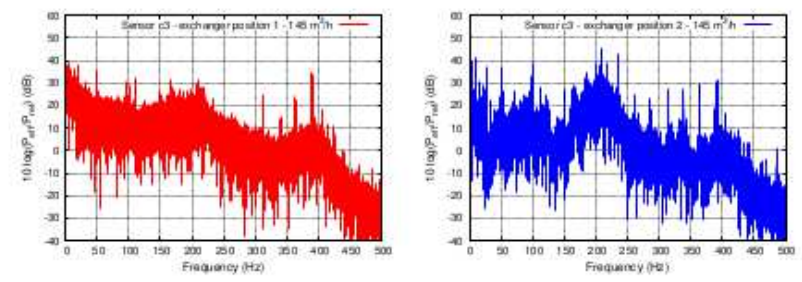

(c) Sensor 3
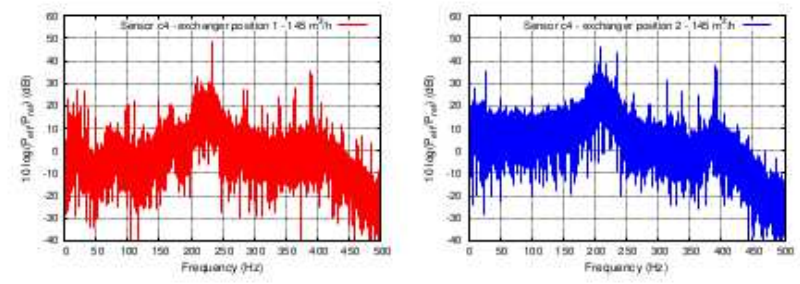

(d) Sensor c4

FIG. 9. Comparison of pressure frequency spectrum for configuration including axial pump and exchanger at $145 \mathrm{~m}^{3} / \mathrm{h}$. $P_{\text {ref }}=1 \mathrm{~Pa}$

axial pump and also for $\mathrm{c} 2$ located downstream the pump but before the exchanger. Taking into account the sensor before and after the exchanger, we can perceive that the exchanger plays the role of an attenuator reducing the pressure amplitudes (see for example sensor c3 for position 1 and $\mathrm{c} 4$ for position 2). Comparing sensor c4, it can be noted that the amplitudes showed reveals the differences because in position 1 of exchanger, $\mathrm{c} 4$ is far of the exchanger discharge and in position 2 of exchanger it is located just off the exchanger.

At $210 \mathrm{~m}^{3} / \mathrm{h}$, the results in figure 10 show that the fluctuations are reduced for sensor $\mathrm{c} 1$ placed before the pump, however the sensors downstream the pump have different behaviors depending of the exchanger position. For example, the fact of moving away the exchanger does not improve the fluctuations rather they worse in sensor c2. The differences in spectra of sensor c3 are due because in position 1 , it is just after the exchanger and in
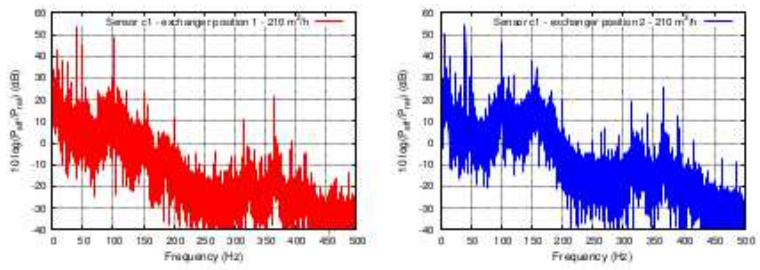

(a) Sensor $\mathrm{c} 1$
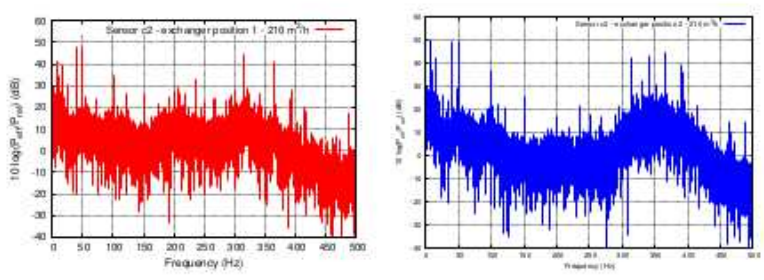

(b) Sensor c2
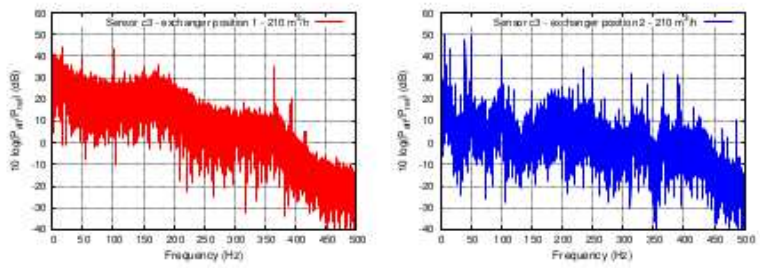

(c) Sensor c3
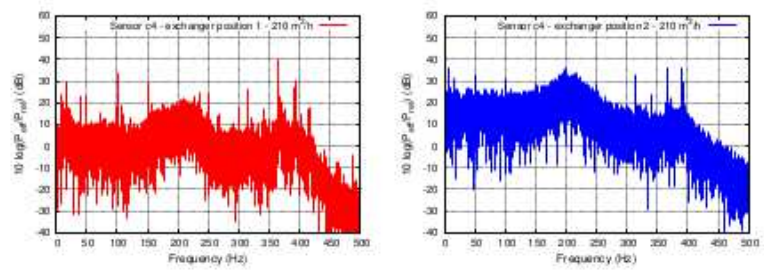

(d) Sensor c4

FIG. 10. Comparison of pressure frequency spectrum for configuration including axial pump and exchanger at $210 \mathrm{~m}^{3} / \mathrm{h}$. $P_{\text {ref }}=1 \mathrm{~Pa}$

position 2, it is before. But in sensor c4, the differences are due to the proximity concerning the exchanger outlet. The amplitudes in position 1 are lower than position 2 because, the fluctuations had more time to dissipate.

Concerning the influence on pressure fluctuations of the configuration including or not the exchanger has been made. In the figure 11, we can see the influence of the exchanger in the pressure field measured by four sensors at $145 \mathrm{~m}^{3} / \mathrm{h}$. For sensor $\mathrm{c} 1$ and $\mathrm{c} 2$, it is clear the influence of the exchanger disturbs and increase the fluctuations being lower when the exchanger is not considered. In relation to the sensors $\mathrm{c} 3$ and $\mathrm{c} 4$, the amplitudes are lower than those when the pump works alone due mainly to the exchanger which plays the role a attenuator.

It can be seen that the spectral analysis shows various peaks at several frequencies related to the rotating frequency and also to blades number and their harmonics. To model the pressure fluctuations, unsteady calculations were necessary. The convergence of these type 

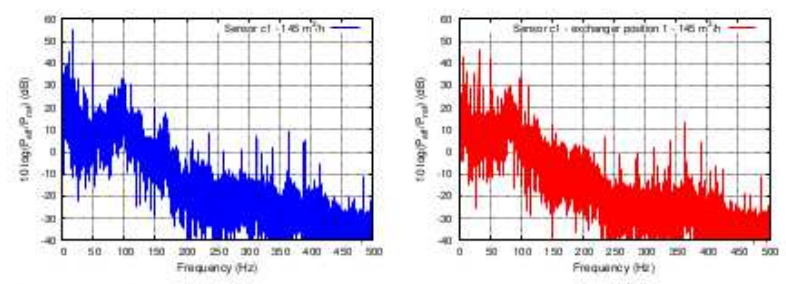

(a) Sensor $\mathrm{c} 1$
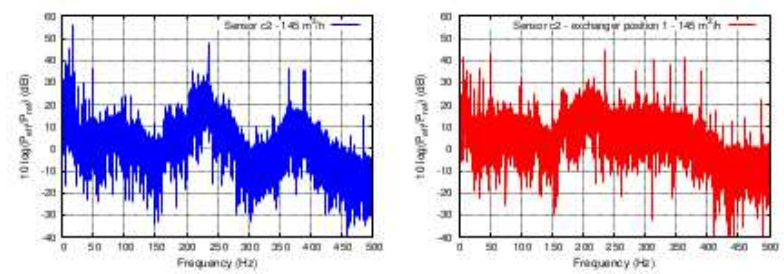

(b) Sensor c2
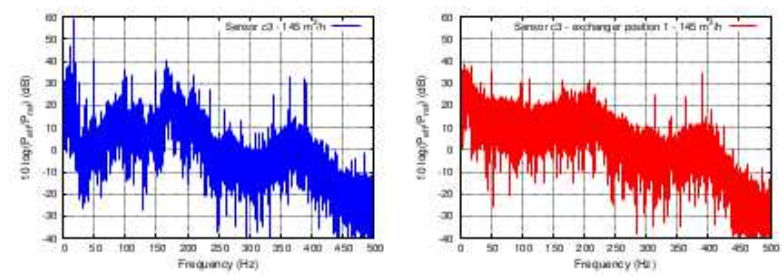

(c) Sensor $\mathrm{c} 3$
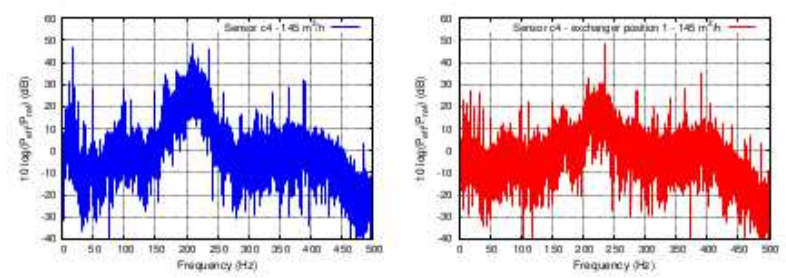

(d) Sensor c4

FIG. 11. Comparison of pressure frequency spectrum for configuration single pump and including axial pump and exchanger at $145 \mathrm{~m}^{3} / \mathrm{h}$. $P_{\text {ref }}=1 \mathrm{~Pa}$
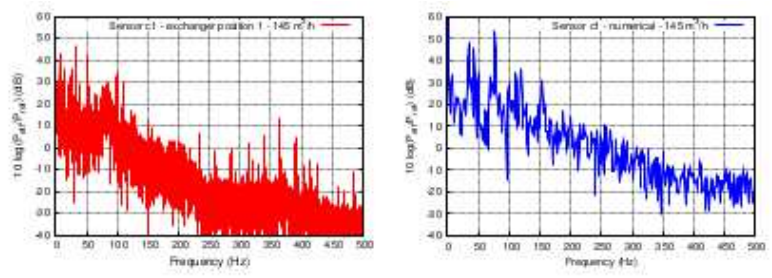

FIG. 12. Numerical and Experimental comparison of the configuration including an axial pump and an exchanger

of calculations is achieved approximately in various rotor revolutions to obtain a periodic unsteady solution specially of the static pressure and also other revolutions to get enough data to have a good frequency resolution concerning the frequency analysis. In this way, the instantaneous static pressure was recorded by virtual sensors placed at the same positions as the test bench. Then the pressure signals were analyzed and processed using the Fast Fourier Transform (FFT) with a Hanning Window as they were in the experimental measurements. The lack of time to let the simulations converge specially for the sensors placed downstream the rotor was an inconvenient to show all the numerical results in order to compare to experimental ones. So in this way, the recorded values of sensor c1 were confronted with the experimental values. The figure 12 illustrate the comparison between numerical and experimental signals, showing the differences at $F_{B P}$ of 8 decibels.

\section{CONCLUSIONS}

Numerical and experimental tests were carried out at DynFluid Laboratory. For this purpose a test bench was built and coupled to the centrifugal pump loop. It was accommodated with several instruments to measure static pressure, electric power and pressure fluctuations. The study concerned a configuration including an axial pump and a bundle of tubes. Four sensors were distributed upstream and downstream the pump. In the numerical simulations, virtual sensors collected the fluctuating pressure signal in the same positions as the experimental ones. The following conclusions can be retained:

i) The experimental nominal point $\left(210 \mathrm{~m}^{3} / \mathrm{h}\right)$ does not match with the numerical one $\left(145 \mathrm{~m}^{3} / \mathrm{h}\right)$.

ii) The highest fluctuations occur upstream the rotor and increase with the presence of the exchanger.

iii) Placing the exchanger after the rotor allows to decrease pressure fluctuations at outlet, but increase those at inlet.

iv) The use of the centrifugal pump to ensure the flow rate to the axial pump modifies the inlet conditions. So, in this way a pressure fluctuations reduction will be detected when the axial pump is fed by the centrifugal one. Further numerical investigations with inlet boundary conditions that mimics this effect would be of great interest.

\section{REFERENCES}

[1] Mitchell, A. B., 1961. "An experimental investigation of cavitation inception in the rotor blade tip region of an axial flow pump". PhD thesis, Aeronautical Research Council.

[2] Laborde, R., Chantrel, P., and Mory, M., 1997. "Tip clearance and tip vortex cavitation in an axial flow pump". Journal Fluids Engineering, 119, pp. 680685.

[3] Horie, C., and Kawaguchi, K., 1959. "Cavitation tests on an axial flow pump". The Japan Society of Mechanical Engineers, 2(5), pp. 187195. 
[4] Kaya, D., 2003. "Experimental study on regaining the tangential velocity energy of axial flow pump". Energy Conversion \& Management, 44, pp. 18171829.

[5] Gao, H., Lin, W., and Du, Z., 2008. "Numerical flow and performance analysis of a water-jet axial flow pump". Ocean Engineering, 35, pp. 16041614.

[6] Saito, S. "The relationship between cavitation development and the velocity and pressure fluctuations of an axial-flow pump". The Japan Society of Mechanical Engineers, 86-1485A, pp. 36823690.

[7] Fu-jun, W., Ling, Z., and Zhi-min, Z., 2007. "Analysis on pressure fluctuation of unsteady flow in axialflow pump". Journal of Hydraulic Engineering, 8, pp. 10031009.

[8] Vad, J., Bencze, F., Benigni, H., Glas, W., and Jaberg, H., 2002. "Comparative investigation on axial flow pump rotors of free vortex and non-free vortex design". Periodica Polytechnica, Ser. Mech. Eng., 46, pp. 107116.

[9] Ida, T., 1970. "Characteristics and flow conditions of a forced-vortex impeller of an axial flow fan:". The Japan Society of Mechanical Engineers, 13(60), pp. 773780 .

[10] Miner, S. M., 1997. "3-d viscous flow analysis of an axial flow pump impeller". International Journal of Rotating Machinery, 3(3), pp. 153161.

[11] De-Sheng, Z., Wei-Dong, S., Bin, C., and XingFan, G., 2009. "Numerical simulation and flow field measurement of high efficiency axial-flow pump". Proceedings of the ASME 2009 Fluids Engineering Division Summer Meeting August 2-6, 2009, Vail, Colorado, USA, pp. 18. 